\title{
Isoquercitrin activates the AMP-activated protein kinase (AMPK) signal pathway in rat H4IIE cells
}

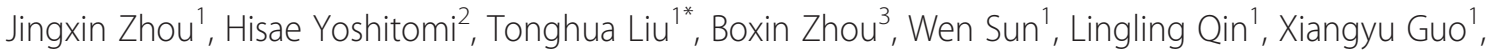
Liansha Huang ${ }^{4}$, Lili Wu ${ }^{1}$ and Ming Gao ${ }^{2^{*}}$

\begin{abstract}
Background: Isoquercitrin, a flavonoid compound that is widely distributed in medicinal and dietary plants, possesses many biological activities, including inhibition of adipocyte differentiation. In this study, we investigated the effect of isoquercitrin on lipid accumulation and its molecular mechanisms in rat hepatoma H4IIE cells.

Methods: To investigate the effect of isoquercitrin on lipid accumulation, H4IIE cells were induced by FFA and the total lipid levels were detected by Oil Red O staining. Furthermore, The protein levels of AMPK and acetyl-CoA carboxylase (ACC), the gene expressions of transcriptional factor, lipogenic genes, and adiponectin receptor 1 (AdipoR1) were analyzed by Western blotting and quantitative real-time PCR. To further confirm the pathway of isoquercitrin-mediated hepatic lipid metabolism, H4lIE cells were treated with an AMPK inhibitor and AdipoR1 siRNA.

Results: Isoquercitrin significantly enhances AMPK phosphorylation, downregulates sterol regulatory element binding protein transcription factor 1 (SREBP-1) and fatty acid synthase (FAS) gene expressions. Pretreatment with AMPK inhibitor, significantly decreased the AMPK phosphorylation and increased FAS expression stimulated by isoquercitrin. Isoquercitrin might also upregulate the expression of AdipoR1 dose-dependently via AMPK in the presence of an AMPK inhibitor and AdipoR1 siRNA.

Conclusions: Isoquercitrin appears to regulate AMPK activation, thereby enhancing AdipoR1 expression, suppressing SREBP-1 and FAS expressions, and resulting in the regulation of lipid accumulation. These results suggest that isoquercitrin is a novel dietary compound that can be potentially be used to prevent lipid metabolic disorder and nonalcoholic fatty liver disease.
\end{abstract}

Keywords: Isoquercitrin, H4IIE cells, AMPK, AdipoR1

\section{Background}

Adenosine monophosphate-activated protein kinase (AMPK) has been recognized as a master regulator of hepatic metabolism. Its activation results in an increase in fatty acid oxidation and suppression of fatty acid synthesis via inactivation of acetyl-CoA carboxylase (ACC). It inhibits the lipogenic gene expression of fatty acid synthase (FAS) by decreasing the action of transcription factors [1]. Sterol regulatory element binding protein 1 (SREBP-1) is the most important transcription factor regulating the expression of lipogenic enzymes, including ACC and FAS.

\footnotetext{
* Correspondence: thliu@vip.163.com; gaoming@mukogawa-u.ac.jp 'Beijing University of Chinese Medicine, 11 North 3rd-ring East Road, Beijing, Chaoyang District 100029, People's Republic of China

${ }^{2}$ School of Pharmaceutical Sciences, Mukogawa Women's University, 11-68 Koshien Kyuban-cho, Nishinomiya, Hyogo 663-8179, Japan

Full list of author information is available at the end of the article
}

Many studies have suggested that increased levels of SREBP-1 are closely associated with fatty liver in obese animal models [2]. Meanwhile, a previous report has shown that SREBP-1 is mediated by AMPK [3]. Therefore, AMPK plays a key role in fatty acid metabolism in the liver and in whole body lipid metabolism.

Lipid metabolic disorder refers to a disruption in lipid metabolism in which there are excessive serum levels of lipids and lipoprotein lipids. This disorder has escalated into a major worldwide public health problem, and it is a critical risk factor for metabolic syndromes, such as insulin resistance, type 2 diabetes mellitus, and cardiovascular diseases [4]. Nonalcoholic fatty liver disease (NAFLD) develops when a large amount of lipid is deposited in hepatocytes, and it is tightly associated with lipid metabolic disorder [5]. Untreated NAFLD may progress to more

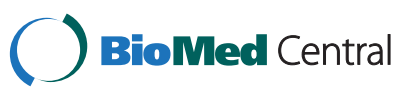


serious diseases, such as liver cirrhosis and hepatocellular carcinoma, and, consequently, influence other body systems to cause atherosclerosis, type 2 diabetes, and hypertension [6]. Therefore, it is essential to identify ways to prevent fatty liver. Treatment of fatty liver focuses on inhibition of excessive free fatty acids (FFAs), either by reducing hepatic lipogenesis or by increasing oxidation or export.

Recently, adiponectin receptors (AdipoRs) and AMPK signaling have been recognized as critical regulators of hepatic lipid metabolism to treat fatty liver. AdipoRs and AMPK signaling suppress fatty acid synthesis by inactivating ACC and FAS and stimulate fatty acid oxidation. SREBP-1, a key lipogenic transcription factor, plays a major role in regulating hepatic lipid metabolism, including lipolysis and fatty acid synthesis [7]. Inhibition of SREBP-1 expression by activating AMPK reduces lipid accumulation in hepatocytes to ameliorate fatty liver. Currently, many pharmaceutical chemicals for the treatment of fatty liver, such as insulin sensitizers and lipidlowering drugs, have the risk of side effects. In contrast, natural dietary compounds, which can ameliorate steatosis, may have fewer risks. These natural compounds also play an important role in improving the quality of life and maintaining health. Therefore, the discovery of food components that can prevent and treat NAFLD is of interest and value.

Isoquercitrin (quercetin3-O-b-D-glucopyranoside) is a natural flavonoid glucoside that is distributed in medicinal and dietary plants, such as vegetables, herbs, and flowers [8]. Isoquercitrin has been found to have a wide range of biological properties, such as anti-inflammatory effects [9]; antioxidant activity, including decreasing ROS levels and reducing lipid peroxidation both in vivo and in vitro [10]; neuroprotection; and promotion of neurite elongation [11]. In particular, a recent study reported that isoquercitrin inhibits adipocyte differentiation of 3 T3-L1 cells via activation of $\mathrm{Wnt} / \beta$-catenin signaling [12]. However, it is not clear whether isoquercitrin mediates AMPK signaling to result in amelioration of lipid metabolism in hepatocytes.

Recently, many studies in cell lines and animal models have examined the ability of food and dietary plant components to prevent fatty liver and hepatic steatosis by mediating hepatic lipid accumulation. Research has focused on luteolin [13] and anthocyanins [14]. Additionally, studies have indicated that isoquercitrin may possess the capability to reduce lipid peroxidation and inhibit adipocyte differentiation. However, it has not been clarified in vitro whether isoquercitrin regulates the hepatic AMPK pathway, which plays a critical role in mediating lipid metabolism and fatty accumulation. In the present study, we investigated the effects of isoquercitrin on the management of the AMPK signaling pathway in rat hepatoma H4IIE cells.

\section{Methods}

\section{Reagents}

Isoquercitrin was purchased from the National Institutes for Food and Drug Control (Beijing, China). Dulbecco's modified Eagle's medium (DMEM) and antibiotic solution was obtained from Nacalai Tesque (Kyoto, Japan). AMPK inhibitor, compound $\mathrm{C}$ was pucharsed from Santa Cruz Biotechnology, Inc. (Texas, USA), and 5-aminoimidazole4-carboxamide 1-ribofuranoside (AICAR) was purchased from Cell Signaling Technology, Inc. (Beverly, USA).

\section{Cell culture}

Rat hepatoma (H4IIE) cells, which were purchased from The Japanese Collection of Research Bioresources Cell Bank (Osaka, Japan), were cultured in Dulbecco's Modified Eagle Medium (DMEM) supplemented with $10 \%$ fetal bovine serum (FBS) and 1\% streptomycin/ penicillin (Nacalai Tesque, Kyoto, Japan) at $37^{\circ} \mathrm{C}$ in a $5 \% \mathrm{CO}_{2}$ humidified atmosphere. Cells at $70-80 \%$ confluence were exposed to various concentrations of isoquercitrin $(50,100$, or $200 \mu \mathrm{M})$ or to $1 \mu \mathrm{L}$ dimethyl sulfoxide (DMSO) as a control for the indicated times. All of the cells were incubated at $37^{\circ} \mathrm{C}$ in a $5 \% \mathrm{CO}_{2}$ humidified atmosphere.

\section{MTT assay}

H4IIE cells were plated at 5000 cells/well in 96-well tissue culture plates. The indicated concentrations of isoquercitrin $(0,50,100$, and $200 \mu \mathrm{M})$ dissolved in DMSO were added and incubated for $72 \mathrm{~h}$. MTT reagent $(0.01 \mathrm{ml}$, $5 \mathrm{mg} / \mathrm{ml}$ ) was added and incubated in each well for $3 \mathrm{~h}$. The MTT medium was replaced with $0.1 \mathrm{ml}$ DMSO, and absorbance was read at $570 \mathrm{~nm}$. Cell viability was calculated by the absorbance of isoquercitrin-treated cells compared to control cells.

\section{Oil Red $\mathrm{O}$ staining and measurement of lipid accumulation}

Oil Red O powder $(150 \mathrm{mg})$ was dissolved in $50 \mathrm{ml}$ of isopropanol to form a stock solution, which was diluted with distilled water to $60 \%$ of the stock concentration. After filtration, the supernatant was stored at room temperature. Subconfluent H4IIE cells were cultured with the control group (DMSO) and the given concentrations of isoquercitrin $(50,100$, or $200 \mu \mathrm{M})$ in DMEM for $24 \mathrm{~h}$. Next, the cells were treated with $0.1 \mathrm{mM}$ FFA for another $24 \mathrm{~h}$ to produce lipid accumulation. The cell layers were washed with phosphate-buffered saline (PBS), fixed in $10 \%$ formalin for $10 \mathrm{~min}$, stained with Oil Red $\mathrm{O}$ dye solution for $20 \mathrm{~min}$ at room temperature, washed with $60 \%$ isopropanol to remove unbound dye, washed with PBS, and then photographed under a microscope. Subsequently, the Oil Red O stain was 
dissolved in isopropanol, and lipid accumulation was measured by absorbance at $540 \mathrm{~nm}$.

\section{Western blotting}

Confluent cells with or without isoquercitrin were prepared with $100 \mu \mathrm{l}$ of ice-cold homogenization buffer containing $50 \mathrm{mM}$ Tris- $\mathrm{HCl}(\mathrm{pH} 7.4), 100 \mathrm{mM} \mathrm{NaCl}$, $1 \%$ Nonidet-P $40,0.25 \%$ sodium deoxycholate, $0.1 \%$ sodium dodecyl sulfate (SDS), $1 \mathrm{mM}$ ethylenediaminetetraacetic acid (EDTA), $50 \mathrm{mM} \mathrm{NaF}, 2 \mathrm{mM} \mathrm{Na}_{3} \mathrm{VO}_{4}, 30 \mathrm{mM}$ sodium pyrophosphate, $2 \mathrm{mM}$ phenylmethanesulfonylfluoride (PMSF), $1 \mathrm{mM}$ benzamidine, $0.02 \mathrm{~g} / \mathrm{mL}$ trypsin inhibitor, $0.02 \mathrm{~g} / \mathrm{mL}$ leupeptin, and $0.02 \mathrm{~g} / \mathrm{mL}$ aprotinin. After incubation on ice for $30 \mathrm{~min}$, the lysates were centrifuged at 12,000 rpm for $10 \mathrm{~min}$, and the supernatants were isolated. Proteins were extracted by boiling in $0.5 \mathrm{mmol} / \mathrm{l}$ Tris-HCl, pH 6.8, 20\% glycerol, 10\% SDS, $0.1 \%$ bromophenol blue, and 10\% 2-mercaptethanol. Equal amounts of protein $(20 \mu \mathrm{g})$ were electrophoresed on a $7.5-12.5 \%$ sodium dodecyl sulfate polyacrylamide gel electrophoresis (SDS-PAGE) gel at $100 \mathrm{~V}$ for $2 \mathrm{~h}$, and transferred onto polyvinylidene fluoride (PVDF) membranes (Amersham Life Science, Inc., Buckinghamshire, UK) at $100 \mathrm{~mA}$ for $2 \mathrm{~h}$. The membranes were blocked in Blocking One-P and Blocking One (Nacalai Tesque, Kyoto, Japan) for $30 \mathrm{~min}$. After blocking, the membranes were incubated overnight at $4^{\circ} \mathrm{C}$ with the following rabbit primary antibodies (at a dilution of 1:1000 in antibody solution 1 [Toyobo, Osaka, Japan]): p-AMPK $\alpha$ Thr172, t-AMPKa, p-ACC Ser79, or t-ACC (Cell Signaling Technology, Beverly, USA). The membranes were then washed with Tris-buffered saline and Tween 20 (TBST) and incubated with horseradish peroxidase-linked anti-rabbit secondary antibodies $(1: 10,000)$ for $1 \mathrm{~h}$ at room temperature. Detection was achieved using Ez Capture ST (ATTO, Tokyo, Japan) with Chemi-Lumi One Super (Nacalai Tesque, Kyoto, Japan). Bands were quantified and scanned using NIH Image. $\beta$-Actin was used as an internal control.

\section{Quantitative real-time PCR}

Total RNA was isolated using Sepasol-RNA I Super G (Nacalai Tesque, Kyoto, Japan). Real-time PCR kits were from TOYOBO (Tokyo, Japan). RNA (1 $\mu \mathrm{g})$ from each sample was reverse-transcribed to cDNA using the ReverTra Ace qPCR RT Kit, according to the manufacturer's instructions (Toyobo). THUNDERBIRD SYBR qPCR Mix was used for quantitative real-time RT-PCR analysis of each gene's expression. The following primers (Invitrogen, California, USA) were used: AdipoR1 forward, TGAGG TACCA GCCAGATGTC; AdipoR1 reverse, CGTGTCC GCT TCTCTGTTAC; FAS forward, GGAACTGAAC GG CATTACTC G; FAS reverse, CATGCCGTTA TCAACTT GTC C; SREBP-1 forward, CCACCCTGTA GGTCACCG
TT T; SPEBP-1 reverse, GTGGGTATAA GCGTTCAGCT GC; $\beta$-actin forward, GGGAAATCGT GCGTGACATT; and $\beta$-actin reverse, GCGGCAGTGG CCATCTC. The amplification was performed as follows: $95^{\circ} \mathrm{C}$ for $1 \mathrm{~min}$ followed by 40 cycles of $95^{\circ} \mathrm{C}$ for $15 \mathrm{~s}, 60^{\circ} \mathrm{C}$ for $32 \mathrm{~s}, 95^{\circ} \mathrm{C}$ for $15 \mathrm{~s}, 60^{\circ} \mathrm{C}$ for $20 \mathrm{~s}$, and $95^{\circ} \mathrm{C}$ for $15 \mathrm{~s}$ with a real-time PCR system (ABI Prism 7000). The data were normalized to $\beta$-actin.

\section{Knockdown with small interfering RNA (siRNA)}

H4IIE cells were seeded in a six-well plate at $2 \times 10^{5}$ cells per well in $2 \mathrm{ml}$ of antibiotic-free normal growth medium supplemented with FBS. Subconfluent cells were transfected with AdipoR1 siRNA or control siRNA (Santa Cruz Biotechnology) in $1 \mathrm{ml}$ Opti-MEM (GIBCO, USA) containing a transfection reagent mixture of Reagent A and B (Santa Cruz Biotechnology, USA) for $7 \mathrm{~h}$. Then, $1 \mathrm{ml}$ of $2 \times$ normal growth medium was added. After a 24-h incubation, the normal growth medium was used for another $48 \mathrm{~h}$ according to the manufacturer's instructions. The transfection efficiency was assessed by qRT-PCR, followed by treatment with isoquercitrin.

\section{Statistical analysis}

Data for individual groups were presented as the mean \pm standard error of the mean (SEM) and were statistically analyzed using Dunnett or Tukey test. Values between two groups were analyzed by Student's $t$-test. Differences were considered significant for $P<0.05$.

\section{Results}

Isoquercitrin inhibits lipid accumulation in FFA-induced H4IIE cells

Cells were cultured with different concentrations of isoquercitrin $(50,100$, and $200 \mu \mathrm{M})$ for $72 \mathrm{~h}$, and cell viability was determined by the MTT assay. As expected, isoquercitrin did not show significant cytotoxic effects (Figure 1A). Thereafter, all indicated doses of isoquercitrin were chosen to investigate the effect of isoquercitrin on lipid accumulation in H4IIE cells. Subconfluent H4IIE cells were incubated in medium containing the indicated concentrations of isoquercitrin for $24 \mathrm{~h}$, followed by adding $0.1 \mathrm{mM}$ FFA for another $24 \mathrm{~h}$. The total lipid levels were detected by Oil Red O staining. As shown in Figure $1 \mathrm{~B}$ and $\mathrm{C}$, isoquercitrin treatment significantly prevented cellular lipid droplet accumulation in H4IIE cells in a dose-dependent manner.

\section{Isoquercitrin increases phosphorylation of AMPKa in H4IIE cells}

To assess the effect of isoquercitrin on phosphorylation of AMPK $\alpha$ that was the possible mechanism responsible for the lipid accumulation, H4IIE cells were treated with isoquercitrin $(50,100$, and $200 \mu \mathrm{M})$ for $12 \mathrm{~h}$. As Thr172 


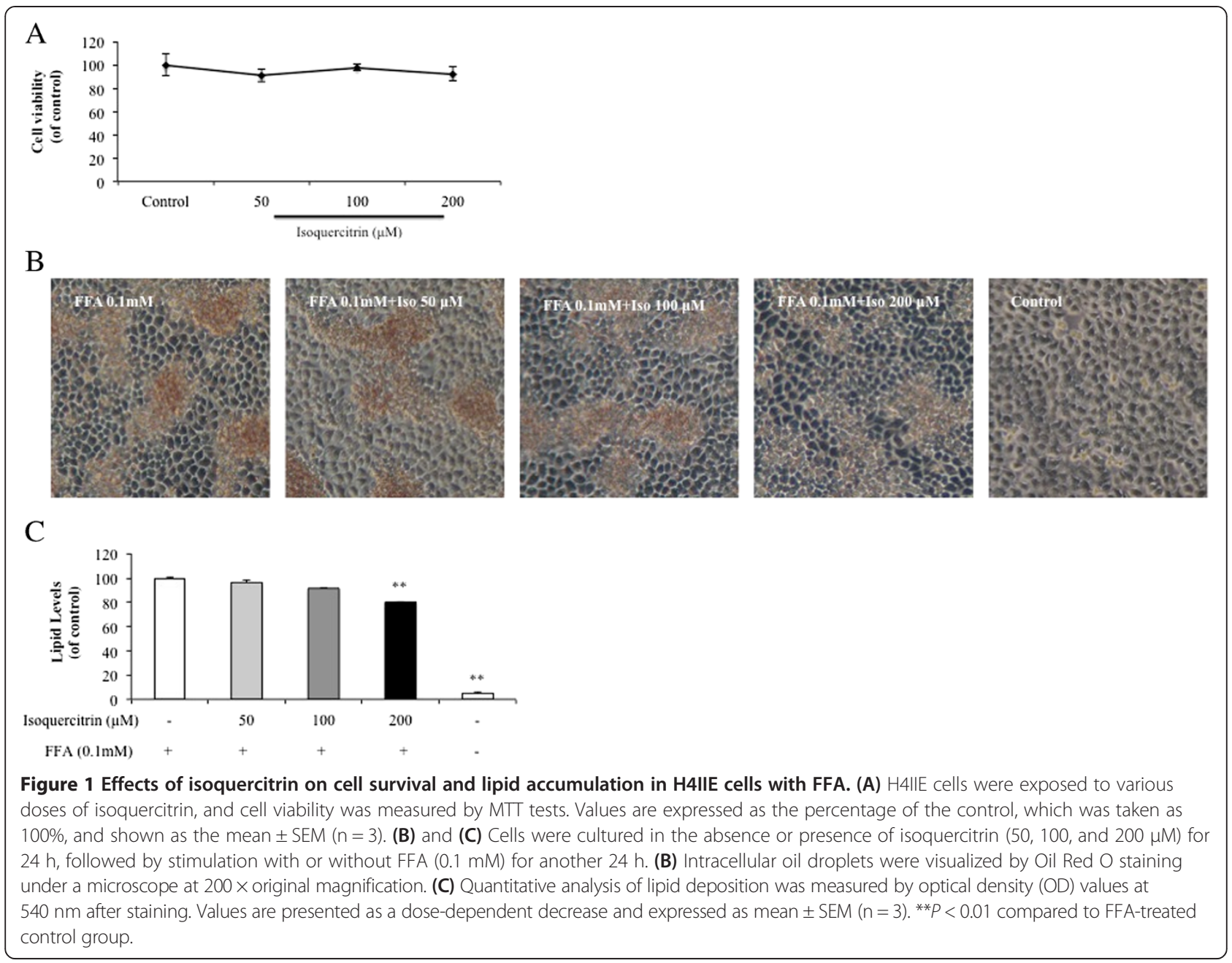

phosphorylation of $\mathrm{AMPK} \alpha$ is an essential marker of AMPK activity, and ACC is its primary downstream targeting enzyme. The phospho-Thr172 antibody of AMPK $\alpha$ and the phospho-Ser79 antibody of ACC were examined by immunoblotting to assess AMPK activation. Isoquercitrin significantly increased the level of pAMPK $\alpha$ over the untreated control, but the expression of endogenous total AMPK $\alpha$ protein showed almost no change (Figure 2A). Moreover, isoquercitrin stimulated phosphorylation of ACC (Ser79), whereas the overall ACC protein expression did not change (Figure 2A). This result indicated that isoquercitrin enhanced the phosphorylation of AMPK and ACC (Figure 2B and C).

\section{Isoquercitrin downregulates SREBP-1 and FAS mRNA expressions in H4IIE cells}

Many reports have shown that AMPK decreases the actions of the transcriptional factor SREBP-1 and suppresses the expression of lipogenic genes, including FAS [15]. Because isoquercitrin enhanced phosphorylation of AMPK and stimulated ACC phosphorylation, the effects of isoquercitrin on the gene expression of SREBP-1 and FAS in H4IIE cells were evaluated by real-time PCR analysis. H4IIE cells treated with isoquercitrin showed significantly decreased SREBP-1 and FAS mRNA expressions (Figure 3A and B).

\section{AMPK pathway regulates isoquercitrin-induced reduction of lipid levels in H4IIE cells}

Because isoquercitrin increased the phosphorylation of AMPK and ACC, and reduced the gene expression levels of SREBP-1 and FAS in H4IIE cells, we investigated the critical role of AMPK signaling on isoquercitrin-mediated hepatic fatty acid metabolism using an AMPK inhibitor, compound C. Pretreatment of compound C significantly decreased the AMPK phosphorylation stimulated by isoquercitrin (Figure 4A). In addition, compound C significantly attenuated the mRNA expression of FAS induced by isoquercitrin in H4IIE cells (Figure 4B). These results indicate that $A M P K$ plays a crucial role in the effect of isoquercitrin in regulating lipid metabolism in H4IIE cells. 

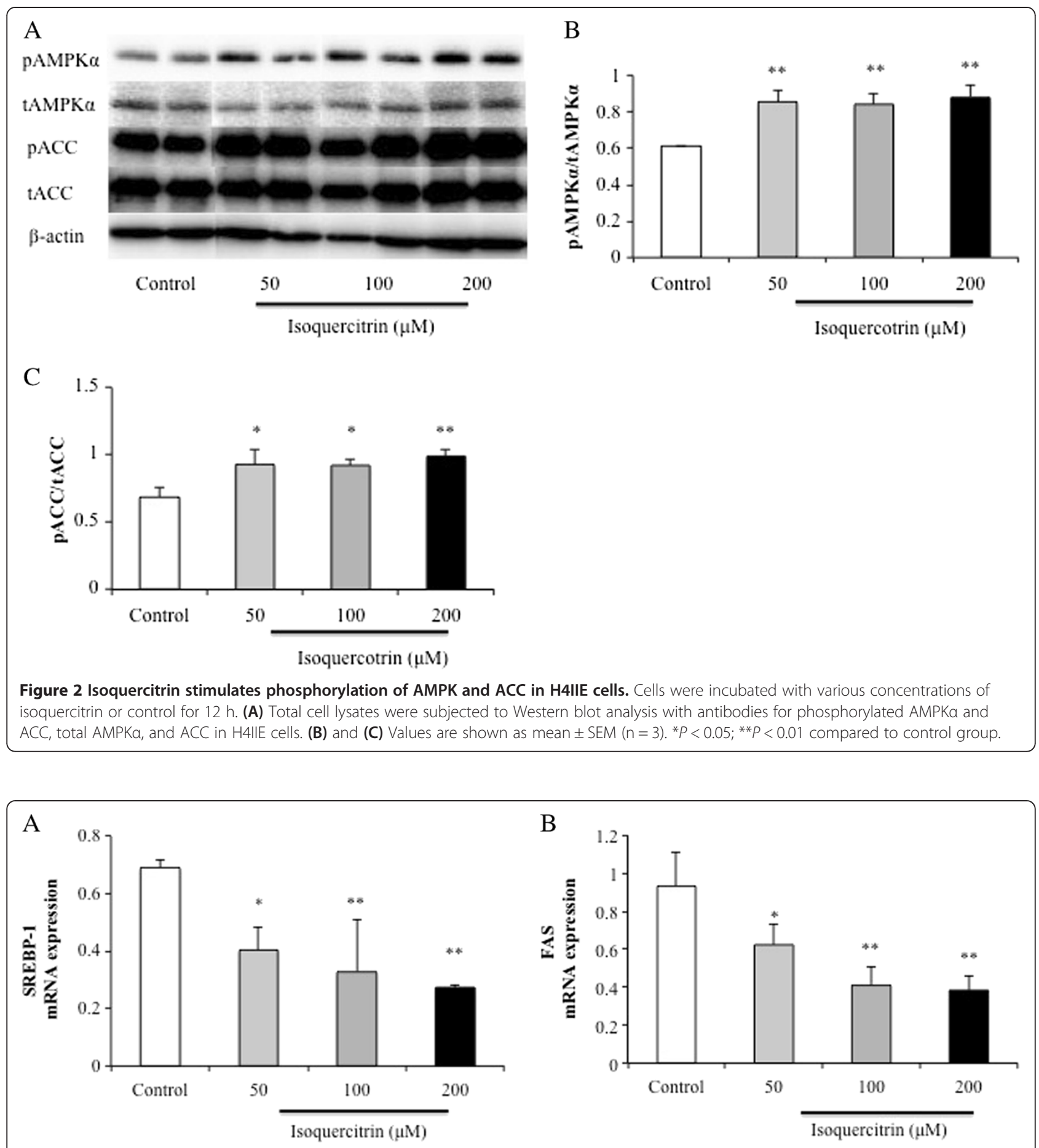

\begin{tabular}{ccc}
\hline Isoquercitrin concentration & SREBP-1 mRNA expression & FAS mRNA expression \\
\hline Control & $0.69 \pm 0.029$ & $0.94 \pm 0.17$ \\
$50 \mu \mathrm{M}$ & $0.40 \pm 0.079^{*}$ & $0.62 \pm 0.11^{*}$ \\
$100 \mu \mathrm{M}$ & $0.33 \pm 0.18^{* *}$ & $0.41 \pm 0.10^{* *}$ \\
$200 \mu \mathrm{M}$ & $0.27 \pm 0.01^{* *}$ & $0.38 \pm 0.08^{* *}$ \\
\hline
\end{tabular}

Figure 3 Isoquercitrin decreases the mRNA expression levels of SREBP-1 and FAS in H4IIE cells. Cells were treated with the indicated concentrations of isoquercitrin for $12 \mathrm{~h}$. The mRNA expression levels of SREBP-1 (A) and FAS (B) were assessed by quantitative real-time PCR. Results are expressed as means \pm SEM $(n=3)$ normalized to $\beta$-actin mRNA expression. ${ }^{*} P<0.05 ;{ }^{* *} P<0.01$ compared to control group. 


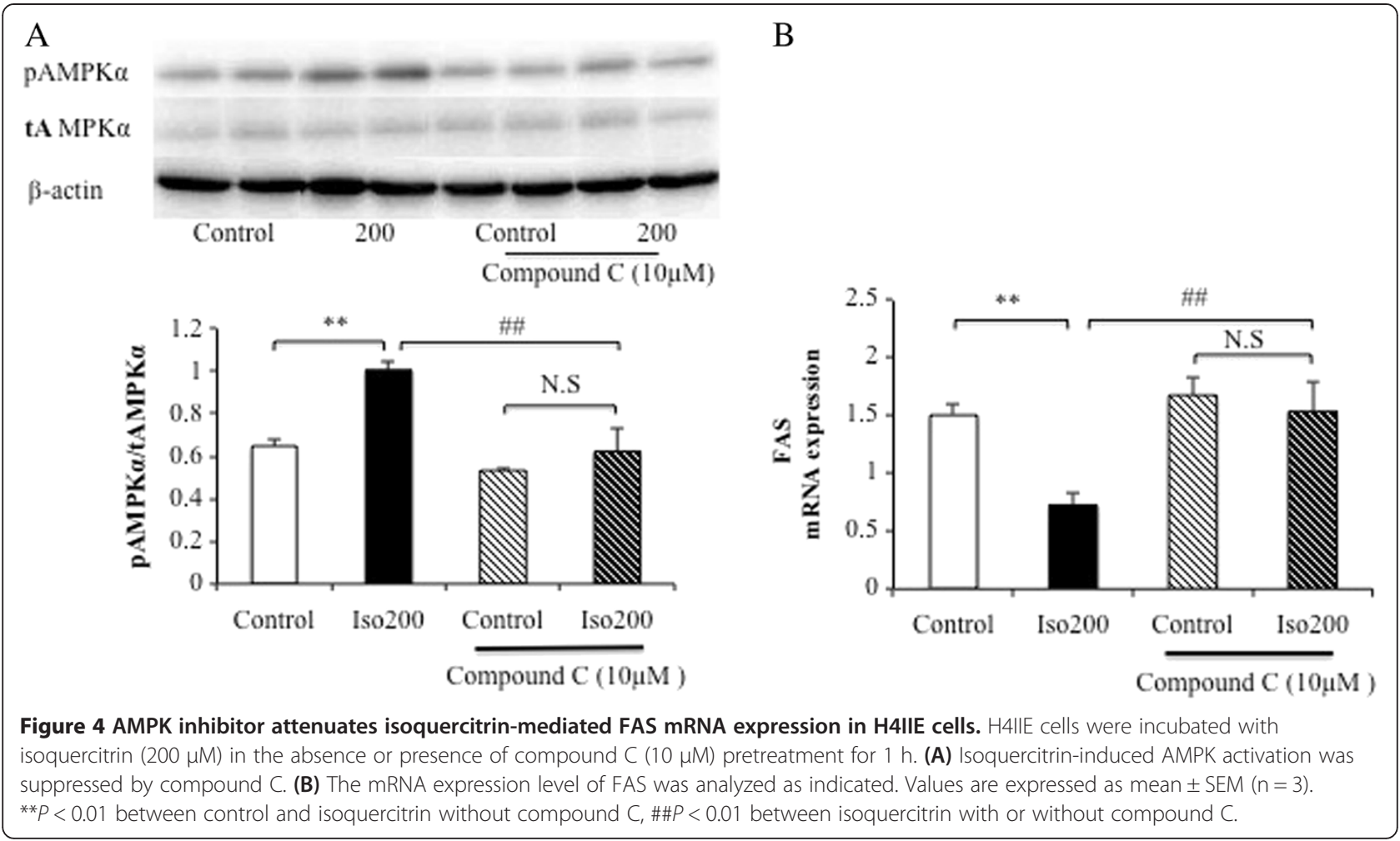

\section{Isoquercitrin upregulates AdipoR1 mRNA expression via AMPK in H4IIE cells}

Next, we studied whether isoquercitrin could regulate AdipoR1 mRNA expression levels in H4IIE cells by using realtime PCR. As expected, AdipoR1 mRNA expression in H4IIE cells was dose-dependently increased by isoquercitrin treatment (Figure 5A). Because isoquercitrin affects both the AdipoR1 mRNA expression level and AMPK phosphorylation, we investigated whether isoquercitrin stimulates the expression of AdipoR1 to result in AMPK activation, or if phosphorylating AMPK leads to an increase in AdipoR1 expression. First, we examined the effect of AMPK phosphorylation mediated by isoquercitrin on AdipoR1 mRNA expression levels by using the AMPK agonist AICAR and the AMPK inhibitor compound C. The results showed that the mRNA expression level of AdipoR1 was significantly increased by AICAR treatment in the H4IIE cells (Figure 5B). Pretreatment of H4IIE cells with compound $\mathrm{C}$ significantly suppressed the isoquercitrin-induced increase in AdipoR1 mRNA expression compared with AICAR treatment, which acted as a positive control (Figure $5 \mathrm{C}$ ). These findings indicated that isoquercitrin might regulate the AdipoR1 mRNA expression level via AMPK.

\section{Isoquercitrin affects AMPK signaling after AdipoR1 knockdown in H4IIE cells}

To evaluate whether isoquercitrin enhances AMPK phosphorylation without regulating AdipoR1, H4IIE cells were transfected with scrambled siRNAs, which served as the control, or with specific siRNAs against AdipoR1. After transfection, AdipoR1 mRNA expression in H4IIE cells was remarkably reduced when compared with the control, suggesting that the knockdown was effective (Figure 6A). The transfection of AdipoR1 siRNA in H4IIE cells did not alter AMPK activation stimulated by isoquercitrin treatment compared with the control group (Figure 6B).

\section{Discussion}

AMPK is a master regulator and a major cellular energy sensor of metabolic homeostasis. Defects in AMPK are associated with metabolic disorders. The increase in the prevalence of lipid metabolic disorders has become a major global public health problem and plays a large role in the subsequent development of NAFLD. Therefore, there has been a worldwide focus on developing prevention methods and therapeutics that target lipid metabolic disorder and NAFLD. Recently, researchers have focused on the clinical effects of dietary compounds from plants, which are known to possess biological properties that reduce the risk of metabolic disorders. Previous in vivo and in vitro studies have suggested that several flavonoids, such as tiliroside, luteolin, and cathechins, act to manage metabolic disorders. Some of the main effects of these dietary compounds are on hepatic lipid metabolism via AMPK activation and FAS gene expression $[13,16,17]$. In the present study, we demonstrate that 

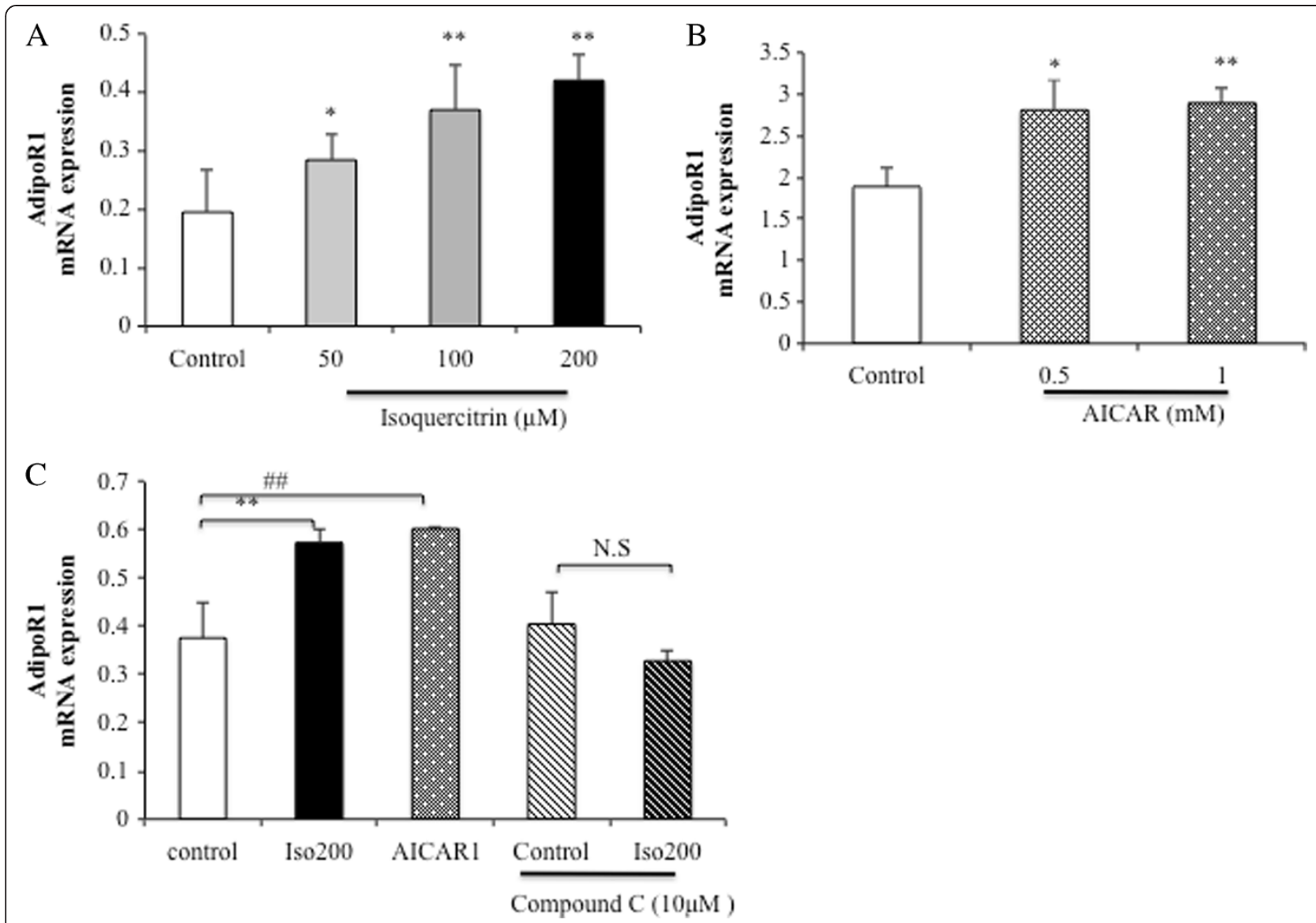

Figure 5 Isoquercitrin increases AdipoR1 mRNA expression levels via AMPK in H4IIE cells. (A) Cells were treated with different concentrations of isoquercitrin as mentioned. (B) H4IIE cells were treated with AICAR ( $0.5 \mathrm{mM}$ and $1 \mathrm{mM})$ for $12 \mathrm{~h}$ as indicated. (C) H4lIE cells were treated with isoquercitrin $(200 \mu \mathrm{M})$ or AICAR $(1 \mathrm{mM})$ for $12 \mathrm{~h}$ in the absence or presence of preincubation with $10 \mu \mathrm{M}$ compound C for $1 \mathrm{~h}$. The mRNA expression levels of AdipoR1 were measured by quantitative real-time PCR and normalized to $\beta$-actin mRNA expression. Values are shown as means $\pm \operatorname{SEM}(n=3)$. (A) ${ }^{*} P<0.05$; ${ }^{*} P<0.01$ compared to control group. (B) ${ }^{*} P<0.05$; ${ }^{*} P<0.01$ compared to control group. (C) ${ }^{* *} P<0.01$ between isoquercitrin and control group, \#\#P<0.01 between AICAR and control group, N.S. between isoquercitrin and isoquercitrin plus compound $C$.

isoquercitrin, a quercetin glucoside, regulates the AMPK pathway to inhibit lipid accumulation in H4IIE cells. Thus, isoquercitrin may be a novel dietary flavonoid that can suppress the development of lipid metabolic disorder and NAFLD.

Numerous studies have indicated that AMPK is a key cellular energy sensor, which plays an important role in regulating the liver and whole-body lipid metabolic homeostasis. In many reports, it has been proved that the extent of phosphorylation of Thr172 reflects the degree of AMPK activation, which is necessary and sufficient for AMPK activity $[18,19]$. The first downstream enzymatic target of AMPK is ACC, which is related to the synthesis of malonyl-CoA. ACC is involved with promoting fatty acid biosynthesis and inhibiting fatty acid oxidation. The suppression of ACC activation by AMPK phosphorylation causes a rate-controlling decrease in malonyl-CoA, followed by a decrease in fatty acid synthesis and an increase in fatty acid oxidation. It also has been reported that phosphorylation of ACC at Ser77 and Ser79 participate in the control of ACC activity [20]. The present study showed that isoquercitrin treatment significantly stimulated AMPK activation and increased the phosphorylation of ACC.

SREBP-1c is a transcription factor, which stimulates fatty acid biosynthesis in the liver by regulating several lipogenic enzymes, such as FAS and ACC. In addition, it has been reported that the expression of SREBP-1 is markedly altered in the liver of obese patients and in the fatty livers of obese mice [2,21]. The action of SREBP-1 is suppressed by AMPK activation and mediates inhibition of ACC and FAS gene expression to regulate liver lipid metabolism in animal models [1]. In addition, some insulin-sensitizing drugs activate AMPK and subsequently, reduce hepatic levels of SREBP-1 and lipogenic gene expression in hepatocytes [3]. 


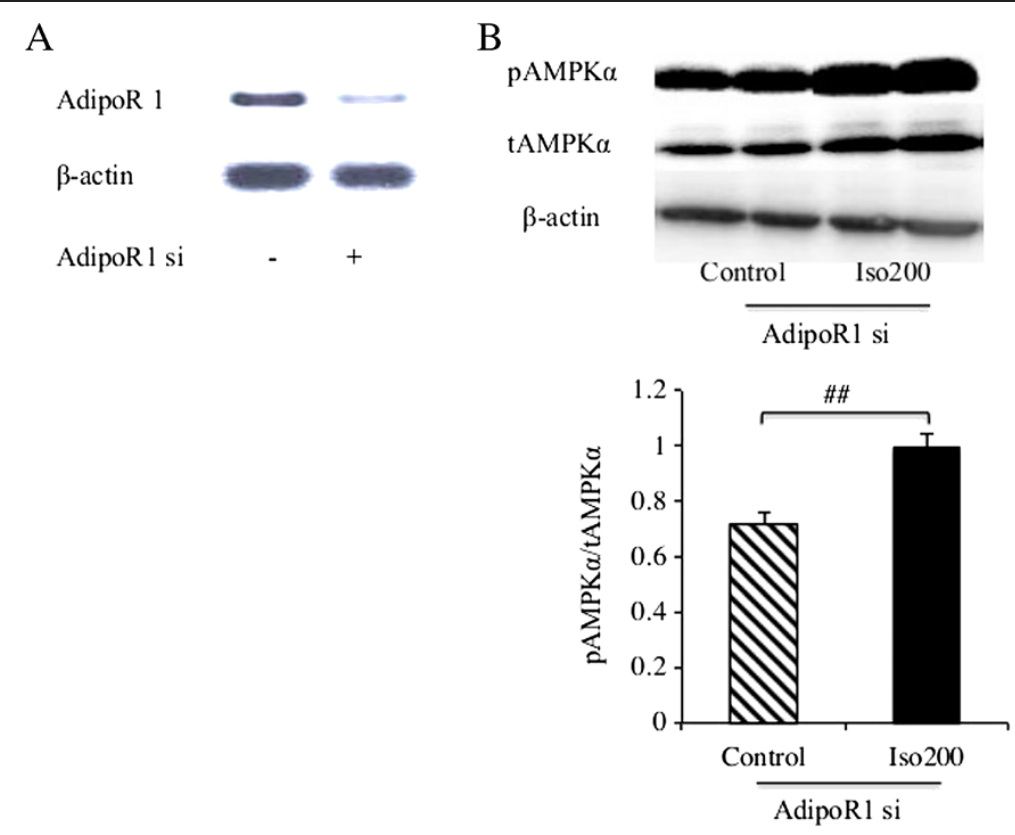

Figure 6 AdipoR1 knockdown does not change isoquercitrin-induced AMPK signaling in H4IIE cells. H4IIE cells were transfected with AdipoR1 siRNA or control siRNA as indicated, followed by isoquercitrin treatment for $12 \mathrm{~h}$. (A) After transfection, AdipoR1 expression was determined by quantitative RT-PCR. (B) Phosphorylation and total AMPKa protein expression were measured by Western blotting. Results were analyzed and shown as means \pm SEM $(n=3)$. \#P<0.05 between isoquercitrin and control plus AdipoR1 siRNA.

Consistent with these reports, our results indicated that isoquercitrin treatment induced a reduction in SREBP-1 mRNA expression levels and a downregulation of its target lipogenic gene FAS in H4IIE cells. Furthermore, pretreatment with compound $C$ reversed the isoquercitrin-mediated decrease in FAS mRNA expression and inhibition of lipid deposition. These observations implied that the enhancement of AMPK activation by isoquercitrin leads to suppression of SREBP-1 and FAS gene expressions, resulting in the isoquercitrininduced reduction in fatty acid accumulation in H4IIE cells.

AdipoR1 and AdipoR2, two major physiological receptors for adiponectin, have been reported to play critical roles in lipid and glucose metabolism [22]. Increasing the expression levels of AdipoR1 and AdipoR2 in the liver of mouse models of obesity and type 2 diabetes can improve insulin resistance and diabetes. Moreover, the disruption of AdipoR1 and AdipoR2 almost completely abolished adiponectin binding in the liver, leading to an increase in triglyceride content, oxidative stress, and inflammation, which resulted in NAFLD and insulin resistance. In addition, it has been reported that the expression levels of AdipoR1 and AdipoR2 are significantly decreased in NAFLD animal models [23]. A recent report showed that disruption of AdipoR1 and AdipoR2 in mice caused the mice to be more vulnerable to insulin resistance than adiponectin-knockout mice. This result indicates that the expressions of AdipoR1 and AdipoR2 may be regulated by more than adiponectin binding and adiponectin actions [22]. AdipoR1 has been found to be involved in activating the AMPK pathway, which mediates lipid metabolism in the liver and regulates globular adiponectin-stimulated AMPK activation in hepatocytes [24]. These studies suggest that increasing AdipoR1 will be a novel prevention approach for metabolism disorders.

Previous studies reported that AdipoR expression is regulated by hormones, cytokines, and metabolism factors, and it is also modified by adiponectin, FFA, the liver $\mathrm{X}$ receptor, and Peroxisome proliferator-activated receptor alpha (PPAR- $\alpha$ ) and peroxisome proliferator-activated receptor gamma (PPAR- $\gamma$ ) agonists [25-27]. Recently, several flavonoids have been reported to regulate the expression of AdipoR1 and AMPK [28,29]. However, it was unknown whether isoquercitrin affects hepatic AdipoR1 mRNA expression and whether AMPK mediates fatty acid metabolism. In this study, we demonstrate that isoquercitrin upregulated the mRNA expression level of AdipoR1 and stimulated AMPK phosphorylation to inhibit lipid accumulation in H4IIE cells.

Many studies have shown that AdipoR1 is tightly related to activating downstream steps in the AMPK pathway to inhibit hepatic fatty acid synthesis and increase fatty acid oxidation. Interestingly, the results of the present study suggest that AMPK activation also increases the mRNA expression level of AdipoR1. The AMPK inhibitor decreased isoquercitrin-induced FAS expression and lipid deposition in H4IIE cells, implying 
that isoquercitrin might be a potent AMPK activator. Meanwhile, isoquercitrin increased AdipoR1 expression without adiponectin stimulation, whereas pretreatment with the AMPK inhibitor attenuated the isoquercitrinmediated increase in AdipoR1 expression levels. In addition, the AMPK agonist AICAR significantly stimulated the mRNA expression level of AdipoR1. Therefore, it seems that isoquercitrin acts as an AMPK activator affecting the expression of AdipoR1, which is consistent with the stimulatory effect of the PPAR $\alpha$ agonist on the regulation of AdipoR2 expression in liver cells [30,31].

Furthermore, our results demonstrate that isoquercitrin activated AMPK compared with the control group in an experiment using transfection of AdipoR1 siRNA in H4IIE cells, which indicates that the main effect of isoquercitrin is on AMPK activation. Thus, by acting as AMPK activator, isoquercitrin may regulate AMPK activation, leading to improve AdipoR1 expression in H4IIE cells. These data show, for what we believe is the first time, that AMPK agonists may increase the mRNA expression level of AdipoR1. This result requires further confirmation in the future.

\section{Conclusions}

The present study indicates the novel finding that isoquercitrin, a dietary flavonoid in herbs, flowers, and vegetables, activates the AMPK signaling pathway in H4IIE cells. Isoquercitrin appeared to stimulate AMPK activation, which enhanced AdipoR1 expression, suppressed SREBP-1 and FAS expressions in H4IIE cells, and resulted in the regulation of fatty acid metabolism. These findings provide cellular molecular evidence demonstrating that isoquercitrin can potentially be used as a novel dietary compound to prevent lipid metabolism disorder and NAFLD. These results need to be investigated in further in vivo studies.

\footnotetext{
Abbreviations

ACC: Acetyl-CoA carboxylase; AdipoR1: Adiponectin receptor 1; AdipoRs: Adiponectin receptors; AMPK: AMP-activated protein kinase; DMEM: Dulbecco's Modified Eagle Medium; DMSO: Dimethyl sulfoxide; FAS: Fatty acid synthase; FFAs: Free fatty acids; NAFLD: Nonalcoholic fatty liver disease; PPAR-a: Peroxisome proliferator-activated receptor alpha; PPAR- - : Peroxisome proliferator-activated receptor gamma; siRNA: Small interfering RNA; SREBP-1: Sterol regulatory element binding protein transcription factor 1.
}

\section{Competing interests}

The authors have declared no financial or commercial competing interest

\section{Authors' contributions}

JZ designed the study, conducted the experiments, and wrote the manuscript. HY performed the experiments, analyzed the data. GM designed the study and wrote the manuscript. WS, LQ, and LW performed the experiments and manuscript preparation. BZ, LH analyzed the data. XG drafted the manuscript. TL provided the initial idea, instructed the study. All authors read and approved of the final manuscript.

\section{Acknowledgements}

This study was supported by grants from the international Scientific Collaborative Project (No. 2010DFB33260) and the key project of Chinese Ministry of Education (No. 311011).

\section{Author details}

${ }^{1}$ Beijing University of Chinese Medicine, 11 North 3rd-ring East Road, Beijing, Chaoyang District 100029, People's Republic of China. ${ }^{2}$ School of

Pharmaceutical Sciences, Mukogawa Women's University, 11-68 Koshien Kyuban-cho, Nishinomiya, Hyogo 663-8179, Japan. 3 Sanshui Hospital affiliated to Guangdong Medical college, 16 Guanghai West Road, Foshan, Guangdong 528000, People's Republic of China. “Shenzhen TCM Hospital, 1 Fuhua Road, Shenzhen, Guangdong, Futian District 518000, People's Republic of China.

Received: 20 August 2013 Accepted: 31 January 2014

Published: 3 February 2014

\section{References}

1. Zhang BB, Zhou G, Li C: AMPK: an emerging drug target for diabetes and the metabolic syndrome. Cell Metab 2009, 9:407-416.

2. Ahmed $\mathrm{MH}$, Byrne $\mathrm{CD}$ : Modulation of sterol regulatory element binding proteins (SREBPs) as potential treatments for non-alcoholic fatty liver disease (NAFLD). Drug Discov Today 2007, 12:740-747.

3. Zhou G, Myers R, Li Y, Chen Y, Shen X, Fenyk-Melody J, Wu M, Ventre J, Doebber T, Fujii N, Musi N, Hirshman MF, Goodyear L, Moller DE: Role of AMP-activated protein kinase in mechanism of metformin action. J Clin Invest 2001, 108:1167-1174.

4. Chen SD, Zhou HH, Lin MT, Li GH, Zhao ZX, Zhang YM: Research of influence and mechanism of combining exercise with diet control on a model of lipid metabolism rat induced by high fat diet. Lipids Health Dis 2013, 12:21.

5. Adams LA, Angulo P, Lindor KD: Nonalcoholic fatty liver disease. CMAJ 2005, 172:899-905.

6. Mendez-Sanchez N, Arrese M, Zamora-Valdes D, Uribe M: Current concepts in the pathogenesis of nonalcoholic fatty liver disease. Liver Int 2007, 27:423-433.

7. Brown MS, Goldstein JL: The SREBP pathway: regulation of cholesterol metabolism by proteolysis of a membrane-bound transcription factor. Cell 1997, 89:331-340.

8. Razavi SM, Zahri S, Zarrini G, Nazemiyeh H, Mohammadi S: Biological activity of quercetin-3-O-glucoside, a known plant flavonoid. Bioorg Khim 2009, 35:414-416.

9. Rogerio AP, Kanashiro A, Fontanari C, da Silva EV, Lucisano-Valim YM, Soares EG, Faccioli LH: Anti-inflammatory activity of quercetin and isoquercitrin in experimental murine allergic asthma. Inflamm Res 2007, 56:402-408.

10. Li R, Yuan C, Dong C, Shuang S, Choi MM: In vivo antioxidative effect of isoquercitrin on cadmium-induced oxidative damage to mouse liver and kidney. Naunyn Schmiedebergs Arch Pharmacol 2011, 383:437-445.

11. Jung SH, Kim BJ, Lee EH, Osborne NN: Isoquercitrin is the most effective antioxidant in the plant Thuja orientalis and able to counteract oxidative-induced damage to a transformed cell line (RGC-5 cells). Neurochem Int 2010, 57:713-721.

12. Lee SH, Kim B, Oh MJ, Yoon J, Kim HY, Lee KJ, Lee JD, Choi KY: Persicaria hydropiper (L.) spach and its flavonoid components, isoquercitrin and isorhamnetin, activate the Wnt/beta-catenin pathway and inhibit adipocyte differentiation of 3 T3-L1 cells. Phytother Res 2011, 25:1629-1635.

13. Liu JF, Ma Y, Wang Y, Du ZY, Shen JK, Peng HL: Reduction of lipid accumulation in HepG2 cells by luteolin is associated with activation of AMPK and mitigation of oxidative stress. Phytother Res 2011, 25:588-596.

14. Guo H, Xia M, Zou T, Ling W, Zhong R, Zhang W: Cyanidin 3-glucoside attenuates obesity-associated insulin resistance and hepatic steatosis in high-fat diet-fed and db/db mice via the transcription factor FoxO1. J Nutr Biochem 2012, 23:349-360.

15. Lee YK, Lee WS, Kim GS, Park OJ: Anthocyanins are novel AMPKalpha1 stimulators that suppress tumor growth by inhibiting mTOR phosphorylation. Oncol Rep 2010, 24:1471-1477.

16. Peluso MR: Flavonoids attenuate cardiovascular disease, inhibit phosphodiesterase, and modulate lipid homeostasis in adipose tissue and liver. Exp Biol Med (Maywood) 2006, 231:1287-1299. 
17. Goto T, Teraminami A, Lee JY, Ohyama K, Funakoshi K, Kim YI, Hirai S, Uemura T, Yu R, Takahashi N, Kawada T: Tiliroside, a glycosidic flavonoid, ameliorates obesity-induced metabolic disorders via activation of adiponectin signaling followed by enhancement of fatty acid oxidation in liver and skeletal muscle in obese-diabetic mice. J Nutr Biochem 2012, 23:768-776.

18. Woods A, Vertommen D, Neumann D, Turk R, Bayliss J, Schlattner U, Wallimann T, Carling D, Rider MH: Identification of phosphorylation sites in AMP-activated protein kinase (AMPK) for upstream AMPK kinases and study of their roles by site-directed mutagenesis. J Biol Chem 2003, 278:28434-42.

19. Stein SC, Woods A, Jones NA, Davison MD, Carling D: The regulation on AMP-actived protein kinase by phosphorylation. Biochem J 2000, 345:437-443.

20. Ha J, Daniel S, Broyles SS, Kim K-H: Critical phosphorylation sites for Acetyl-CoA Carboxylase Activity. J Biol Chem 1994, 269:22162-68.

21. Pettinelli P, Del Pozo T, Araya J, Rodrigo R, Araya AV, Smok G, Csendes A Gutierrez L, Rojas J, Korn O, Maluenda F, Diaz JC, Rencoret G, Braghetto I, Castillo J, Poniachik J, Videla LA: Enhancement in liver SREBP-1C/PPARalpha ratio and steatosis in obese patients: correlations with insulin resistance and $n-3$ long-chain polyunsaturated fatty acid depletion. Biochim Biophys Acta 2009, 1792:1080-1086.

22. Yamauchi T, Nio Y, Maki T, Kobayashi M, Takazawa T, I wabu M, Okada-Iwabu M, Kawamoto S, Kubota N, Kubota T, Ito Y, Kamon J, Tsuchida A, Kumagai K, Kozono H, Hada Y, Ogata H, Tokuyama K, Tsunoda M, Ide T, Murakami K, Awazawa M, Takamoto I, Froguel P, Hara K, Tobe K, Nagai R, Ueki K, Kadowaki T: Targeted disruption of AdipoR1 and AdipoR2 causes abrogation of adiponectin binding and metabolic actions. Nat Med 2007, 13:332-339.

23. Ogasawara M, Hirose A, Ono M, Aritake K, Nozaki Y, Takahashi M, Okamoto N, Sakamoto S, Iwasaki S, Asanuma T, Taniguchi T, Urade Y, Onishi S, Saibara $T$, Oben JA: A novel and comprehensive mouse model of human non-alcoholic steatohepatitis with the full range of dysmetabolic and histological abnormalities induced by gold thioglucose and a high-fat diet. Liver Int 2011, 31:542-551.

24. Awazawa M, Ueki K, Inabe K, Yamauchi T, Kaneko K, Okazaki Y, Bardeesy N, Ohnishi S, Nagai R, Kadowaki T: Adiponectin suppresses hepatic SREBP1c expression in an AdipoR1/LKB1/AMPK dependent pathway. Biochem Biophys Res Commun 2009, 382:51-56.

25. Inukai K, Nakashima Y, Watanabe M, Takata N, Sawa T, Kurihara S, Awata T, Katayama S: Regulation of adiponectin receptor gene expression in diabetic mice. Am J Physiol Endocrinol Metab 2005, 288:E876-882.

26. Kharroubi I, Rasschaert J, Eizirik DL, Cnop M: Expression of adiponectin receptors in pancreatic beta cells. Biochem Biophys Res Commun 2003, 312:1118-1122.

27. Chinetti G, Zawadski C, Fruchart JC, Staels B: Expression of adiponectin receptors in human macrophages and regulation by agonists of the nuclear receptors PPARalpha, PPARgamma, and LXR. Biochem Biophys Res Commun 2004, 314:151-158.

28. Ahn TG, Yang G, Lee HM, Kim MD, Choi HY, Park KS, Lee SD, Kook YB, An HJ: Molecular mechanisms underlying the anti-obesity potential of prunetin, an 0-methylated isoflavone. Biochem Pharmacol 2013, 85:1525-1533.

29. Takikawa M, Inoue S, Horio F, Tsuda T: Dietary anthocyanin-rich bilberry extract ameliorates hyperglycemia and insulin sensitivity via activation of AMP-activated protein kinase in diabetic mice. J Nutr 2010, 140:527-533.

30. Tsuchida A, Yamauchi T, Takekawa S, Hada Y, Ito Y, Maki T, Kadowaki T: Peroxisome proliferator-activated receptor (PPAR) alpha activation increases adiponectin receptors and reduces obesity-related inflammation in adipose tissue: comparison of activation of PPARalpha, PPARgamma, and their combination. Diabetes 2005, 54:3358-3370.

31. Sun $X$, Han R, Wang Z, Chen Y: Regulation of adiponectin receptors in hepatocytes by the peroxisome proliferator-activated receptor-gamma agonist rosiglitazone. Diabetologia 2006, 49:1303-1310.

doi:10.1186/1472-6882-14-42

Cite this article as: Zhou et al:: Isoquercitrin activates the AMP-activated protein kinase (AMPK) signal pathway in rat H4IIE cells. BMC

Complementary and Alternative Medicine 2014 14:42.

\section{Submit your next manuscript to BioMed Central and take full advantage of:}

- Convenient online submission

- Thorough peer review

- No space constraints or color figure charges

- Immediate publication on acceptance

- Inclusion in PubMed, CAS, Scopus and Google Scholar

- Research which is freely available for redistribution 